\title{
650.
}

\section{ON A QUARTIC SURFACE WITH TWELVE NODES.}

[From the Quarterly Journal of Pure and Applied Mathematics, vol. xIV. (1877), pp. 103-106.]

WRITE for shortness

$$
\begin{array}{lll}
a=\beta-\gamma, & f=\alpha-\delta, & a f=p, \\
b=\gamma-\alpha, & g=\beta-\delta, & b g=q, \\
c=\alpha-\beta, & h=\gamma-\delta, & c h=r ;
\end{array}
$$

then, $\theta$ being a variable parameter, the surface in question is the envelope of the quadric surface

$$
(\alpha+\theta)^{2} a g h X^{2}+(\beta+\theta)^{2} b h f Y^{2}+(\gamma+\theta)^{2} c f g Z^{2}+(\delta+\theta)^{2} a b c W^{2}=0
$$

viz. this is

$$
\Sigma \alpha^{2} a g h X^{2} \cdot \Sigma a g h X^{2}-\Sigma \alpha a g h X^{2}=0
$$

There are no terms in $X^{4}$, \&c.; the coefficient of $Y^{2} Z^{2}$ is

$$
\gamma^{2} c f g \cdot b f h+\beta^{2} b f h \cdot c f g-2 \beta b f h \cdot \gamma c f g
$$

which is

$$
=b c f^{2} g h(\beta-\gamma)^{2},=a^{2} b c f^{2} g h,=a b c f g h \cdot p .
$$

Hence the whole equation divides by $a b c f g h$, and throwing out this factor, the result is

$$
p\left(Y^{2} Z^{2}+X^{2} W^{2}\right)+q\left(Z^{2} X^{2}+Y^{2} W^{2}\right)+r\left(X^{2} Y^{2}+Z^{2} W^{2}\right)=0
$$

or, observing that $p+q+r=0$, this may also be written

$$
p(Y Z+X W)^{2}+q(Z X+Y W)^{2}+r(X Y+Z W)^{2}=0
$$


and also

$$
p(Y Z-X W)^{2}+q(Z X-Y W)^{2}+r(X Y-Z W)^{2}=0 .
$$

The more general equation

$$
(p, q, r, l, m, n \chi Y Z+X W, Z X+Y W, X Y+Z W)^{2}=0
$$

represents a quartic surface (octadic) having the 8 nodes

We have

$$
\begin{array}{ll}
(1,0,0,0), & (\overline{1}, 1,1,1), \\
(0,1,0,0), & (1, \overline{1}, 1,1), \\
(0,0,1,0), & (1,1, \overline{1}, 1), \\
(0,0,0,1), & (1,1,1, \overline{1}) .
\end{array}
$$

$$
\begin{aligned}
& d_{X} U= \\
& \text { p. } X W^{2}+Y Z W \\
& \text { q. } Y W^{2}+Y Z W \\
& \text { r. } Z W^{2}+Y Z W \\
& \text { l. } 2 X Y Z+W\left(Y^{2}+Z^{2}\right) \\
& \text { m. } 2 X Y W+Z\left(W^{2}+Y^{2}\right) \\
& \text { n. } 2 X Z W+Y\left(W^{2}+Z^{2}\right) \text {, } \\
& d_{Y} U= \\
& \text { p. } Y Z^{2}+X Z W \\
& \text { q. } Y W^{2}+X Z W \\
& \text { r. } Y X^{2}+X Z W \\
& \text { l. } 2 X Y W+Z\left(W^{2}+X^{2}\right) \\
& \text { m. } 2 Y Z X+W\left(Z^{2}+X^{2}\right) \\
& \text { n. } 2 Y Z W+X\left(W^{2}+Z^{2}\right) \text {, } \\
& d_{Z} U= \\
& \text { p. } Y^{2} Z+X Y W \\
& \text { q. } X^{2} Z+X Y W \\
& \text { r. } W^{2} Z+X Y W \\
& \text { l. } 2 Z X W+Y\left(W^{2}+X^{2}\right) \\
& \text { m. } 2 Y Z W+X\left(W^{2}+Y^{2}\right) \\
& \text { n. } 2 Z X Y+W\left(X^{2}+Y^{2}\right) \text {, } \\
& d_{W} U= \\
& \text { p. } \quad X^{2} W+X Y Z \\
& \text { q. } Y^{2} W+X Y Z \\
& \text { r. } Z^{2} W+X Y Z \\
& \text { l. } 2 W Y Z+X\left(Y^{2}+Z^{2}\right) \\
& \text { m. } 2 W Z X+Y\left(Z^{2}+X^{2}\right) \\
& \text { n. } 2 W X Y+Z\left(X^{2}+Y^{2}\right) \text {. }
\end{aligned}
$$

Hence there will be a node

$$
\begin{aligned}
& \mathbf{1}, \overline{\mathbf{1}}, \overline{\mathbf{1}}, 1, \text { if } p+q+r+2 l-2 m-2 n=0, \\
& \overline{\mathbf{1}}, \mathbf{1}, \overline{\mathbf{1}}, 1, \ldots p+q+r-2 l+2 m-2 n=0, \\
& \overline{\mathbf{1}}, \overline{\mathbf{1}}, 1,1, \ldots p+q+r-2 l-2 m+2 n=0, \\
& 1,1,1,1, \ldots p+q+r+2 l+2 m+2 n=0
\end{aligned}
$$

or say there will be

1 of these nodes if $p+q+r+2 l+2 m+2 n=0$,

$$
\begin{aligned}
& 2 \text {................. } p+q+r+2 l=0, m+n=0 \text {, } \\
& 3 \text {............... } p+q+r=2 l=-2 m=-2 n \text {, } \\
& 4 \text {.................. } p+q+r=0, l=0, m=0, n=0 \text {; }
\end{aligned}
$$


viz. the surface having the 12 nodes is the original surface

where

$$
p(Y Z+X W)^{2}+q(Z X+Y W)^{2}+r(X Y+Z W)^{2},
$$

The Jacobian of the quadrics

$$
p+q+r=0
$$

$$
Y Z+X W=0, Z X+Y W=0, X Y+Z W=0,
$$

is

$$
\left\|\begin{array}{cccc}
W, & Z, & Y, & X \\
Z, & W, & X, & Y \\
Y, & X, & W, & Z
\end{array}\right\|=0
$$

viz. the equations are

$$
\begin{aligned}
& X^{3}-X\left(Y^{2}+Z^{2}+W^{2}\right)+2 Y Z W=0 \\
& Y^{3}-Y\left(Z^{2}+X^{2}+W^{2}\right)+2 Z X W=0 \\
& Z^{3}-Z\left(X^{2}+Y^{2}+W^{2}\right)+2 X Y W=0 \\
& W^{3}-W\left(X^{2}+Y^{2}+Z^{2}\right)+2 X Y W=0
\end{aligned}
$$

each of which is satisfied in virtue of any one of the pairs of equations

$$
\begin{array}{c|c}
(Y-Z=0, X-W=0) & (Y+Z=0, X+W=0) \\
(Z-X=0, Y-W=0) & (Z+X=0, Y+W=0) \\
(X-Y=0, Z-W=0) & (X+Y=0, Z+W=0)
\end{array}
$$

so that the Jacobian curve is, in fact, the six lines represented by these equations.

Any two of the three tetrads form an octad, the 8 points of intersection of three quadric surfaces; a figure representing the relation of the 12 points to each other may be constructed without difficulty.

Each tetrad is a sibi-conjugate tetrad quoad the quadric $X^{2}+Y^{2}+Z^{2}+W^{2}=0$. The three tetrads are not on the same quadric surface. 